\title{
Calibration of a seismic hazard assessment tool using velocity fields and geotechnical data
}

\author{
Y Abolfazlzadeh ESG Solutions, Canada \\ L Smith-Boughner ESG Solutions, Canada
}

Z Anderson ESG Solutions, Canada

A Jalbout Goldcorp Inc., Canada

A Mataseje ESG Solutions, Canada

\begin{abstract}
The majority of seismic hazard assessment tools are solely based on statistical analyses of several seismic source parameters such as event rate and time, and seismic moment. These analyses are often applied to the entire mining area which can impact the accuracy and reliability of the hazard assessment tool in each zone. Experience has shown that mining geomechanical risk is complex and its mitigation needs a broad understanding of other geotechnical factors such as rock mass properties, geological structures, mining method, stress regime, etc. Since all the contributing parameters and their impact are not entirely understood, it is critical to apply a range of geotechnical/geomechanical analyses in correlation to each other and quantify the changes in the rock mass behaviour. The goal of this paper is to develop a seismic hazard assessment tool calibrated for each geotechnical domain within the mine. To develop the tool, we incorporated mine geotechnical and geological data, seismic source parameters, and tomography analyses from a hard rock underground mine in North America. There exist several sub-vertical faults and one horizontal structure in the mine which create clear contrasts in rock mass behaviour across the structure. The results show good correlation among the different datasets, and a calibrated seismic hazard tool has been developed that provides ongoing updates to the mine operation.
\end{abstract}

Keywords: seismic source parameters, apparent stress trends, velocity fields, seismic hazard assessment

\section{Introduction}

The monitoring of microseismic activity in mines is an essential practice to ensuring the safety of mining operations and personnel. Real-time warnings and historical information assist in the short- and long-term mining decisions taken by operators. Seismically active mines rely on microseismic data to keep mining safely. The analysis and interpretation of the data recorded by microseismic systems requires a multi-pronged approach to motivate informed decision-making in practice. In operations with complex rock mass response conditions, an approach utilising many lenses for insight on microseismic trends helps contribute to establishing robust risk-assessment models. By testing these models iteratively against past observations, additional confidence can be obtained and meaning extracted in the context of future best practices or preventative measures.

Historically, many deterministic and probabilistic methods have been established to find the precursors of large events to mitigate the risk. These methods are mainly based on the Gutenberg-Richter distribution (Woodward \& Tierney 2017; Wesseloo 2014) or seismic source parameters such as seismic work, strain rate, spatial clustering (Malek \& Leslie 2006; Vallejos \& McKinnon 2011), apparent stress ratio (Brown \& Hudyma 2017), and energy index (Young 2012; Carusone \& Hudyma 2017).

This paper presents a calibrated seismic hazard assessment procedure, supported by mine geotechnical data, seismic source parameters and velocity fields. 


\section{2 Éléonore mine background}

The Éléonore mine (Figure 1) is located about $800 \mathrm{~km}$ north of Montreal and $200 \mathrm{~km}$ east of James Bay in the northeastern part of the Opinaca Reservoir in Quebec. The site is accessed by air from various cities in Quebec and by all-season-road for the transportation of supplies. Exploration in the mine area extends as far back as 1964 with Goldcorp Inc. taking full control in 2006. Further development of the mine has since led to construction starting in 2010 with the first gold pour achieved on 1 October 2014. The Opinaca Reservoir overlies the orebody (Roberto deposit). The underground infrastructure is accessed via the main production shaft (reaches 1,190 m below surface), a ventilation shaft (reaches $725 \mathrm{~m}$ below surface and used to be the exploration shaft), and a $4.5 \mathrm{~km}$ long ramp located on the hanging wall side of the orebody, with the portal located about $300 \mathrm{~m}$ from the shaft.

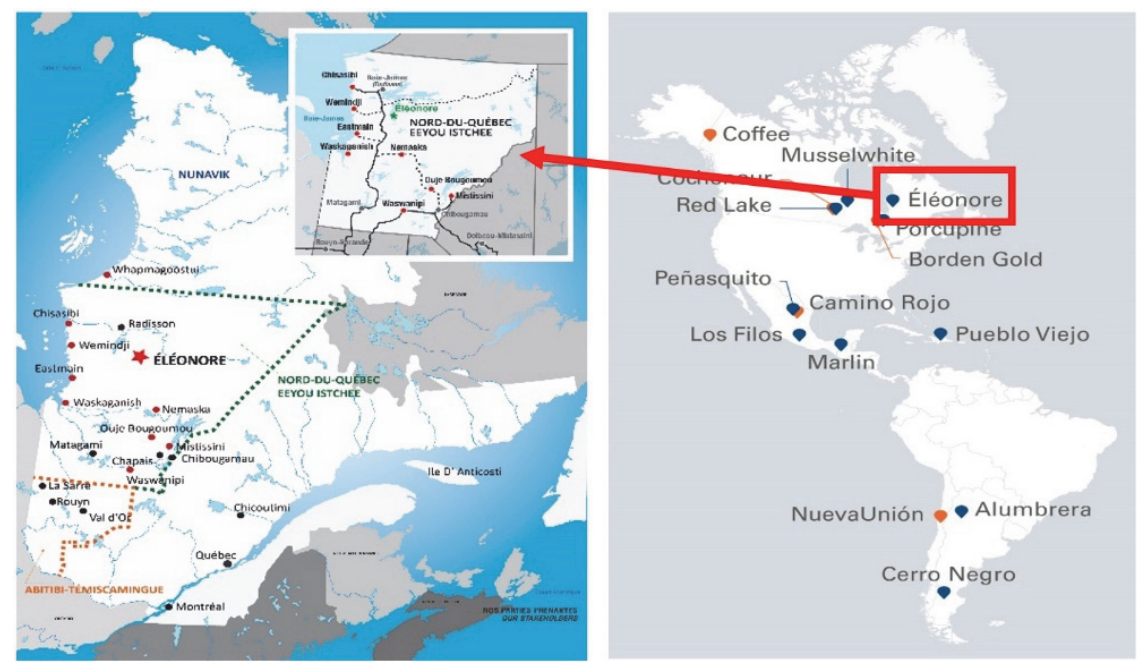

Figure 1 Location of Éléonore mine

The mine is situated in the contact zone between the La Grande and Opinaca subprovinces of the Superior Province (Goldcorp Inc. 2012), entails mining of the Roberto gold deposit. The crescent-shaped orebody (disseminated and stockwork mineralisation) is hosted in meta-sedimentary rocks (turbiditic greywacke and paragneiss) of La Grande Subprovince (Goldcorp Inc. 2012; Ravenelle et al. 2010). Gold mineralisation, traced to a depth of $1,500 \mathrm{~m}$, is found in a series of subparallel zones. Regionally, the Opinaca Subprovince is composed of meta-sedimentary rocks intruded by granodiorite and granitic pegmatite (Ravenelle et al. 2010). The La Grande Subprovince, which surrounds the Opinaca Subprovince on its west and north sides (Goldcorp Inc. 2012), consists of volcanic and meta-sedimentary rocks intruded by tonalite, granodiorite, and diorite (Ravenelle et al. 2010). The first stope production was in July 2014 and the commercial production started on October 2015. The average daily production will reach about 7,000 tpd in 2019. Presently, the mine uses the open stoping method from three principal ore lenses, and the main production is from five active horizons, and the sixth horizon will be coming into production late 2019.

\section{Analysis of seismic source parameters}

Routine seismic source parameters analysis can unveil trends in rock mass changes and can be used as an input for mining design purposes. Therefore, three seismic source parameters were selected to be studied in this paper:

1. $E_{s} / E_{p}$ : the ratio of the $S$-wave energy to the $P$-wave energy is strongly related to the seismic source mechanism (Dahlen 1974). Scale dependence, or the influence of event size, is a critical consideration in analysing seismic source mechanisms. It is important to realise that the smaller and larger events could have different seismic source mechanisms. Case studies on Big Bell fault at Big Bell (Hudyma 2008) and Telfer Gold (Abolfazlzadeh \& Hudyma 2016) mines in Australia (both in high stress regime) showed that the smaller events can have high $E_{s} / E_{p}$ values. 
2. Static stress drop: for seismic events related to fault slip, the static stress drop $(\Delta \sigma)$ is defined as the average difference between the initial and final (shear) stresses (Brune 1970):

$$
\Delta \sigma=\frac{7 M_{o}}{16 r_{o}^{3}}
$$

where:

$$
\begin{aligned}
& \Delta \sigma=\text { static stress drop. } \\
& M_{O}=\text { seismic moment. } \\
& r_{O}=\text { source radius }(\mathrm{m}) .
\end{aligned}
$$

3. Apparent stress: is the amount of stress that the rock mass experiences prior to failure. Variations in apparent stress indicate different failure processes, often due to differences in rock mass characteristics (Wyss \& Brune 1986):

$$
\sigma_{a}=\frac{\mu E}{M_{o}}
$$

where:

$$
\begin{aligned}
& \sigma_{a}=\text { apparent stress. } \\
& \mu \quad=\text { modulus of rigidity. } \\
& E \quad=\text { seismic energy. } \\
& M_{O} \quad=\text { seismic moment. }
\end{aligned}
$$

\subsection{Changes in the seismic source parameters}

Based on the latest mine site investigation report (Mine Design Engineering 2017), the scanline mapping identified two structural domains; the upper and lower domains. The transition between these domains occurs around horizon 4 (Figure 2). This transition may be related to the large sub-horizontal structure that intersects 770 Level. The upper domain has a dominant sub-horizontal set, and the lower domain has a dominant south-dipping sub-vertical set (foliation parallel jointing).

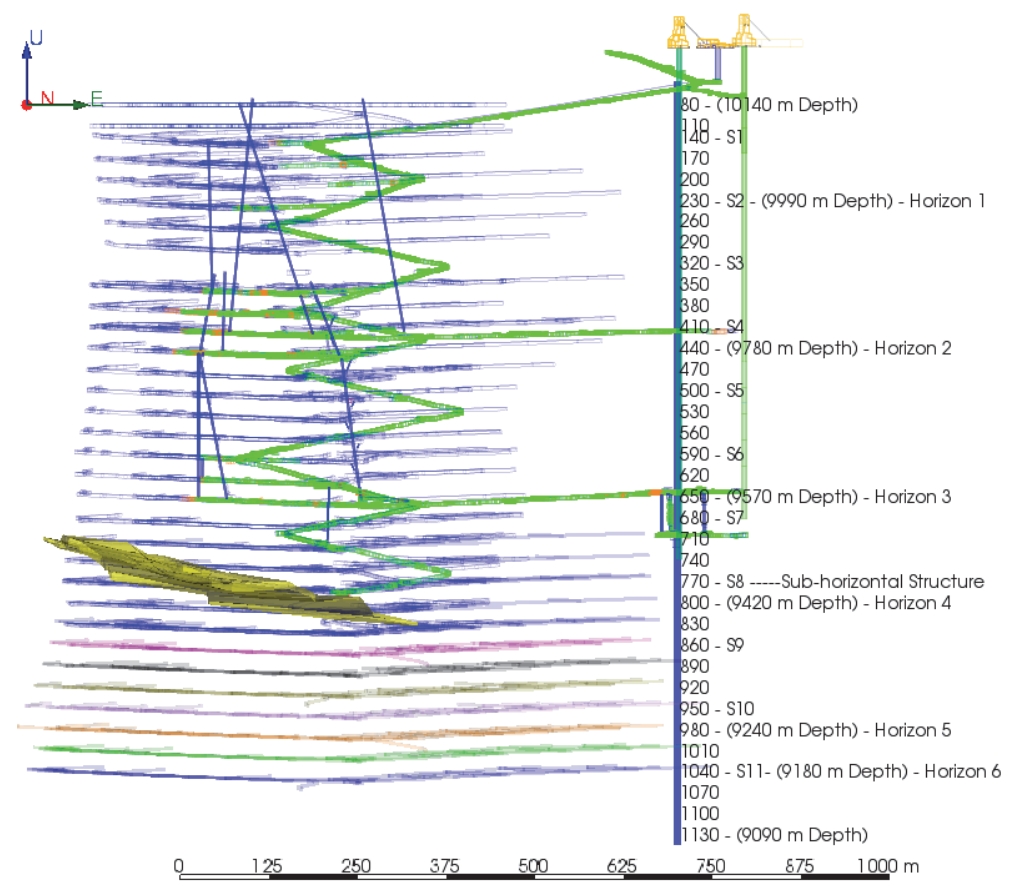

Figure 2 Éléonore mine layout, different levels (e.g. 80), horizons (e.g. horizon 1) and sections (e.g. S1), location of the sub-horizontal structure (yellow plane), looking north 
As Figure 3 elucidates, recorded seismic events from 2015 to mid-2018 were plotted according to the depth versus moment magnitude, and their seismic source parameters. The seismic source parameters showed their expected values (i.e. higher $E_{s} / E_{p}$ for high $M_{w}$ ). However, below a certain depth, low magnitude events represent higher values of $E_{s} / E_{p}$, and static stress drop (apparent stress will be discussed in detail later). This is an indication of rock mass behaviour change, and it matches with mine site investigation results.

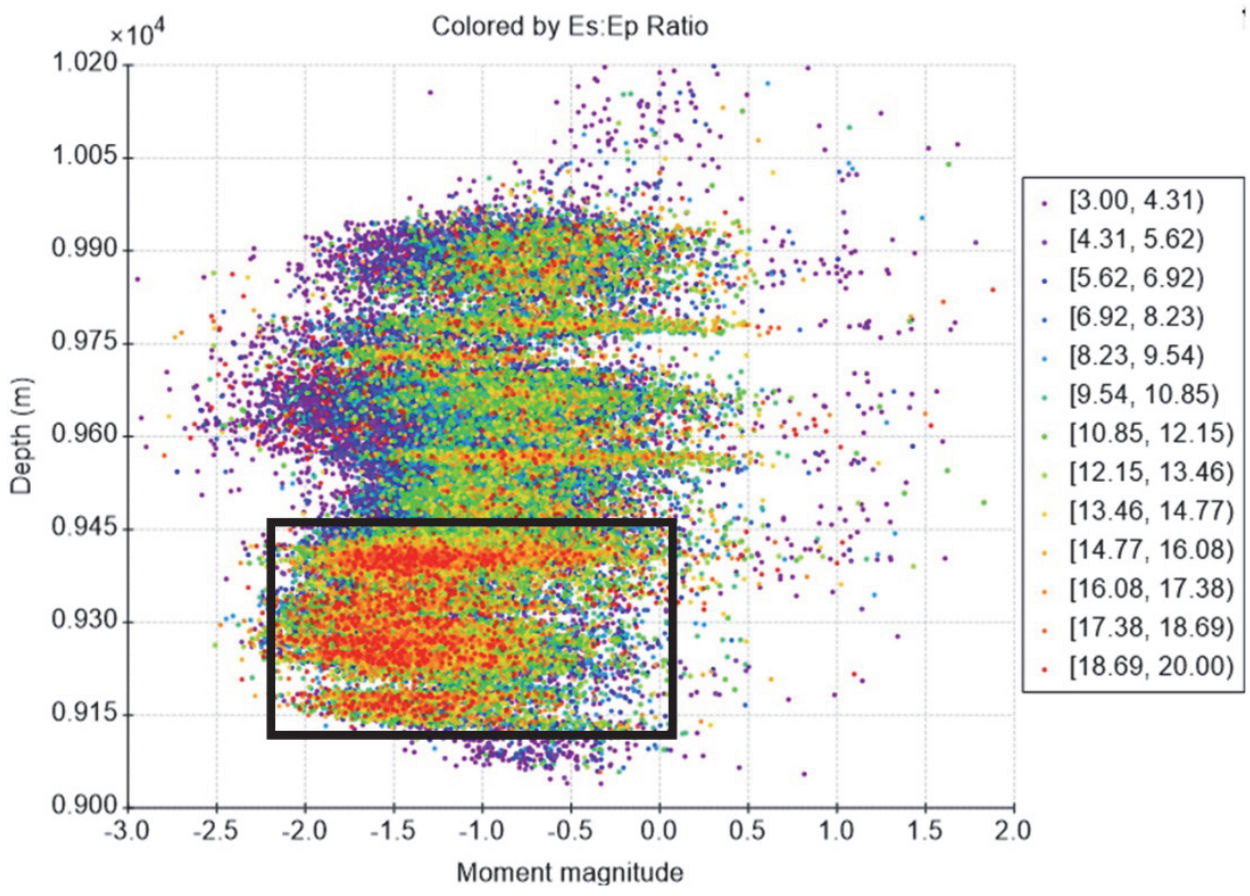

(a)

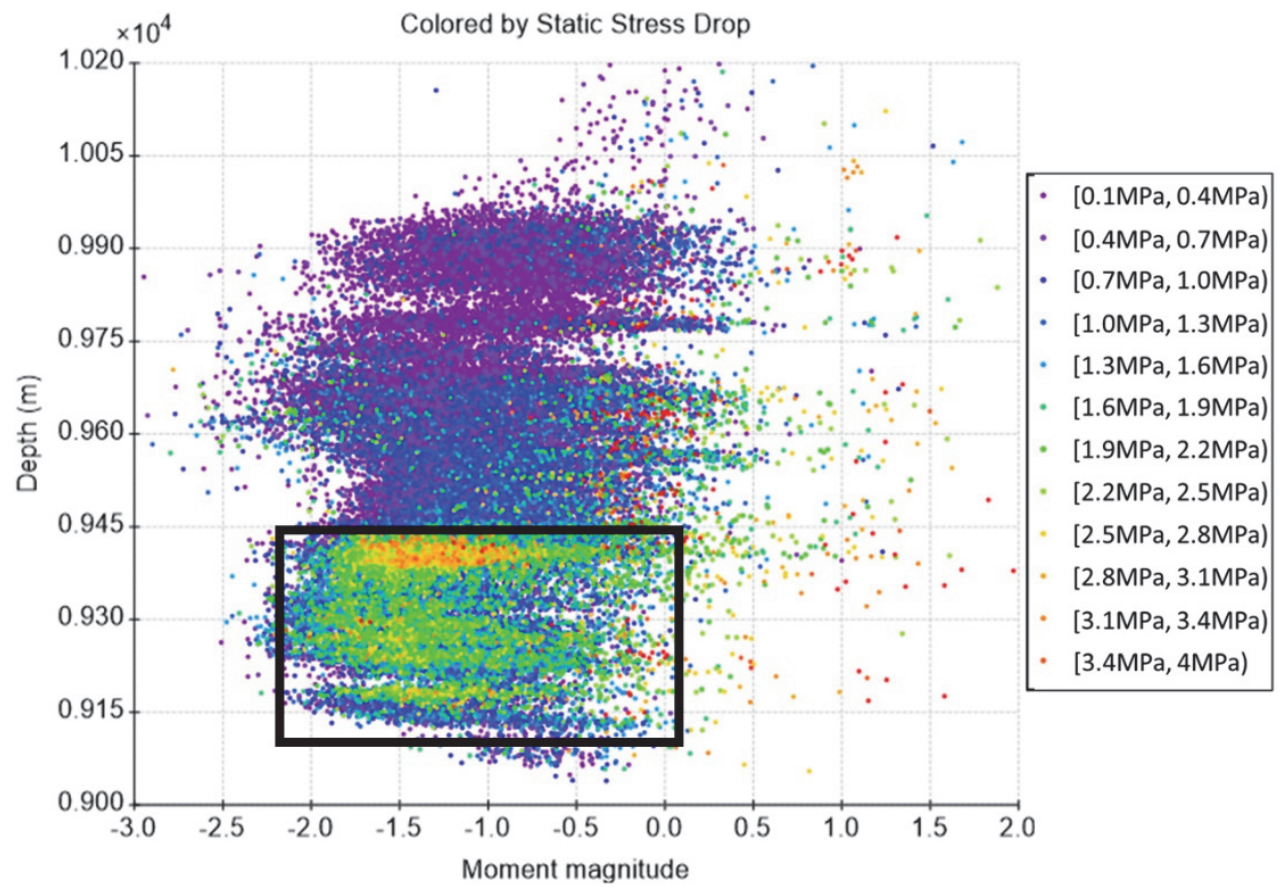

(b)

Figure 3 Seismic events plotted according to the depth and their source parameters. (a) $E_{s} / E_{p i}$ (b) Static stress drop. Rectangles show the locations of lower magnitude events with higher source parameter values 


\subsection{Cumulative apparent stress trends}

The apparent stress scale dependency is an ongoing controversial discussion. Beside the need for having a complete seismic catalogue, the state of stress which is acting on the rock should be considered. In a high-stress regime with a high degree of jointing, it is possible to record low magnitude events with high apparent stress along the joints. For example, in Éléonore mine, a significant change in apparent stress is noticed below a certain elevation. It is also an abnormal trend for low-magnitude events to show high apparent stress. This indicates significant change in the stress status of the rock mass compared to the regions above it.

The area between the top and bottom of the mine is divided into $90 \mathrm{~m}$ sections (i.e. Section 2 includes events above 230L and under 140L); a total of 11 sections, to investigate the cumulative apparent stress (CAS) trends (Figure 2). This procedure was conducted for both blasts and events. The motivation was to explore the consistency between blasts and event trends. Éléonore mine uses a similar blast type (emulsion) in different levels, and the CAS trend of the blasts show an obvious increasing trend in depth. Figure 4 shows the aforementioned trends from top to bottom of the mine. For consistency in the comparison between the blasts and events, the first two sections were removed from study due to lack of event numbers for plotting the CAS. The third section has the distribution on the far left, and Section 11 has the distribution on the far right. This could be an indication of an increase in the stress gradient from surface to depth. It is important to note that these trends are time-independent, which means, although the blasts were recorded at different levels during different time periods, they still show an increasing pattern in their CAS trends in depth. In contrast, some levels showed identical CAS distributions, implying these regions have similar stress regimes.

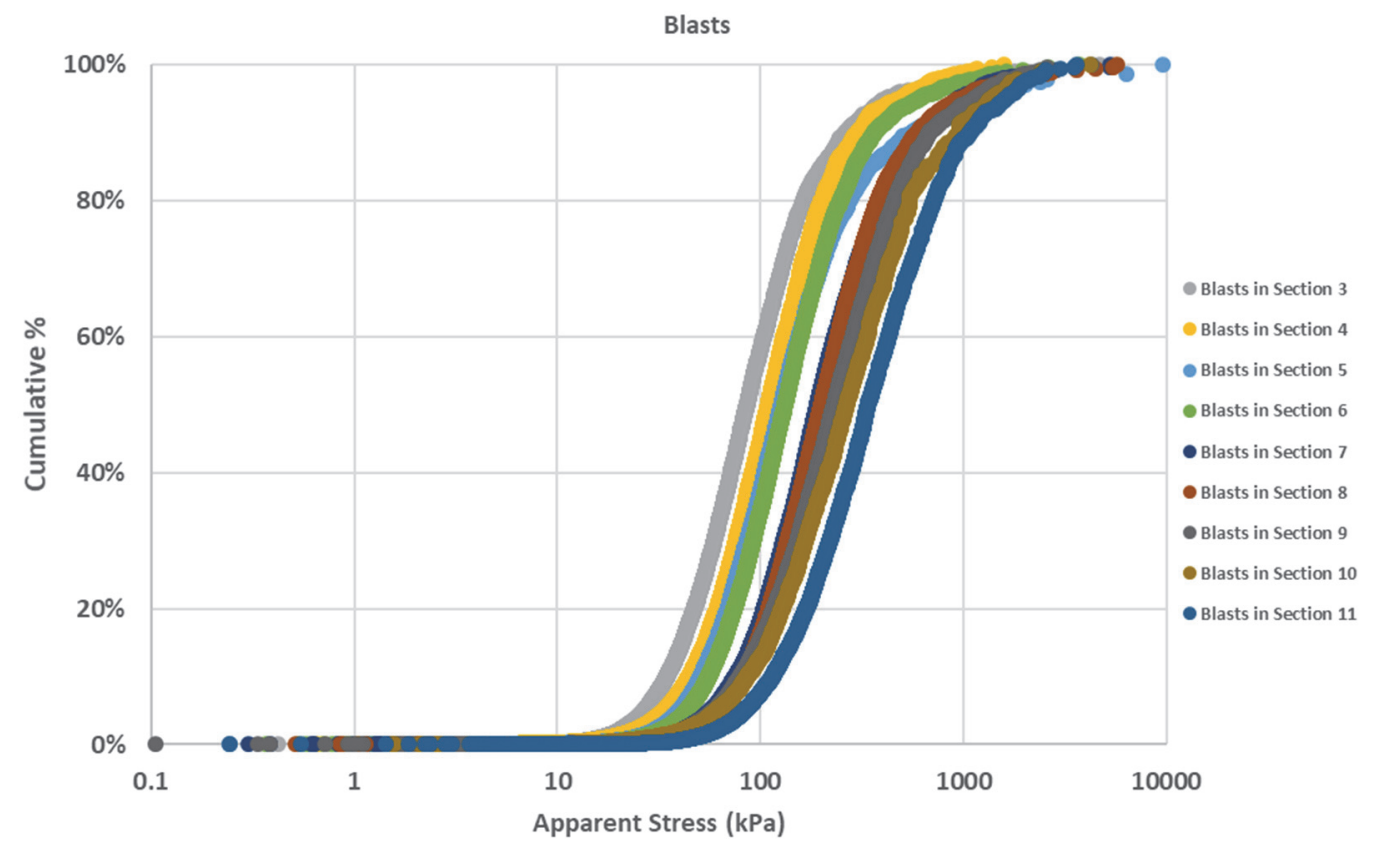

Figure 4 Cumulative apparent stress of blasts in different sections

To delve deeper into the analysis, blasts $\left(-1.5<\mathrm{M}_{\mathrm{w}}<2\right)$ and events $\left(-1.5<\mathrm{M}_{\mathrm{w}}<2\right)$ were plotted according to their moment magnitude scale, but similar results were found. Moreover, a jump in the events' CAS could be an indication of the change in the rock mass properties or stress. For example, when the events' CAS trend reaches to the location of the large sub-horizontal structure, a big jump can be seen in the distributions. This behaviour is more apparent in the events with lower moment magnitudes $\left(-1.5<M_{w}<-1\right)$ between Sections 8 and 9 in the region under the big sub-horizontal structure (Figure 5). This is the area where a change in the $E_{s} / E_{p}$ and static stress drop was observed too. 


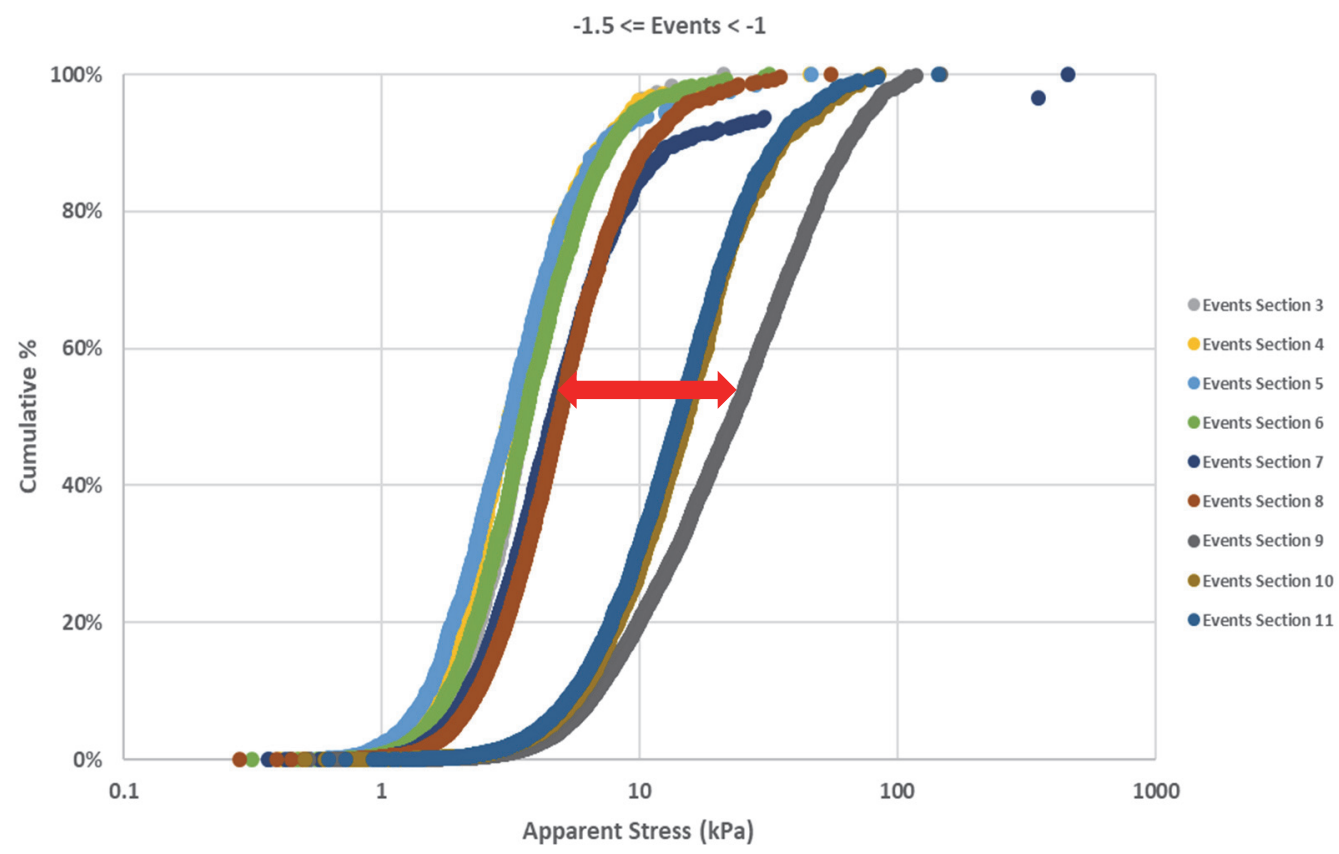

Figure 5 Cumulative apparent stress of events in different sections Red arrow shows the jump between Sections 8 and 9

\section{Tomography}

Seismic tomography relies on recorded seismic data to evaluate stress and strain changes in the rock mass. The advantage of tomography is that it samples regions of the rock mass that may not directly experience seismic activity. The method does so by assessing intersections of ray paths from events/blasts to sensors and measuring the changes in the $\mathrm{P}$ - and $\mathrm{S}$-wave velocities. Using the velocities of the $\mathrm{V}_{\mathrm{p}}$ as a proxy for stress, $V_{s}$ as a proxy to rock stiffness, and $V_{p} / V_{s}$ as a proxy for Poisson's ratio. Higher values of $V_{p} / V_{s}$ correspond to a higher Poisson's ratio (more ductile), and vice versa.

As a starting point for tomography, the mining area is discretised into a grid of 3D volume elements and the events clustered by the volume in which they happen. The velocity model used for the analysis consists of a 1D background model (used to locate the events) and a 3D velocity perturbation. Blasts and events with known location and times are used as inputs for a grid of volume elements as obtained through travel-time tomography. Continued tracking of velocity changes uses the ray paths of subsequent blast and events. For a better resolution, it is necessary to have volumes with numerous crossing ray paths from different directions, but this is not always the case. In other words, different locations of the mine may have different resolutions based on activity rate and sensor distribution. At the time the data used in this paper were acquired, the central part of the mine was well-covered by the array, providing a dense crossing of rays providing for a robust determination of velocities in these regions. To understand the effects of resolution imposed by the ray geometry on the calculated velocities more succinctly, a checkerboard test is used (see Rawlinson \& Sambridge 2003 for a discussion of this methodology). The test consists of a synthetic model of travel times with alternating blocks of increasing and decreasing velocities. When these synthetic travel times are inverted, areas that recover the input synthetic model are said to be well resolved, and the smearing effects of poorly constraining ray geometry is also observed in the recovered images. To further test the robustness of the inversions, random arrival time picking errors are added to the input travel times.

Results of the tomography code for four consecutive weeks (mid-June to mid-July 2018) showed a clear contrast in $\mathrm{V}_{\mathrm{p}} / \mathrm{V}_{\mathrm{s}}$ above and below the sub-horizontal structure (another match with geotechnical domains and seismic source parameters). This variation in the $V_{p} / V_{s}$ ratio suggests that the Poisson's ratio is decreasing below the sub-horizontal structure and could be slightly more brittle (Figure 6). 

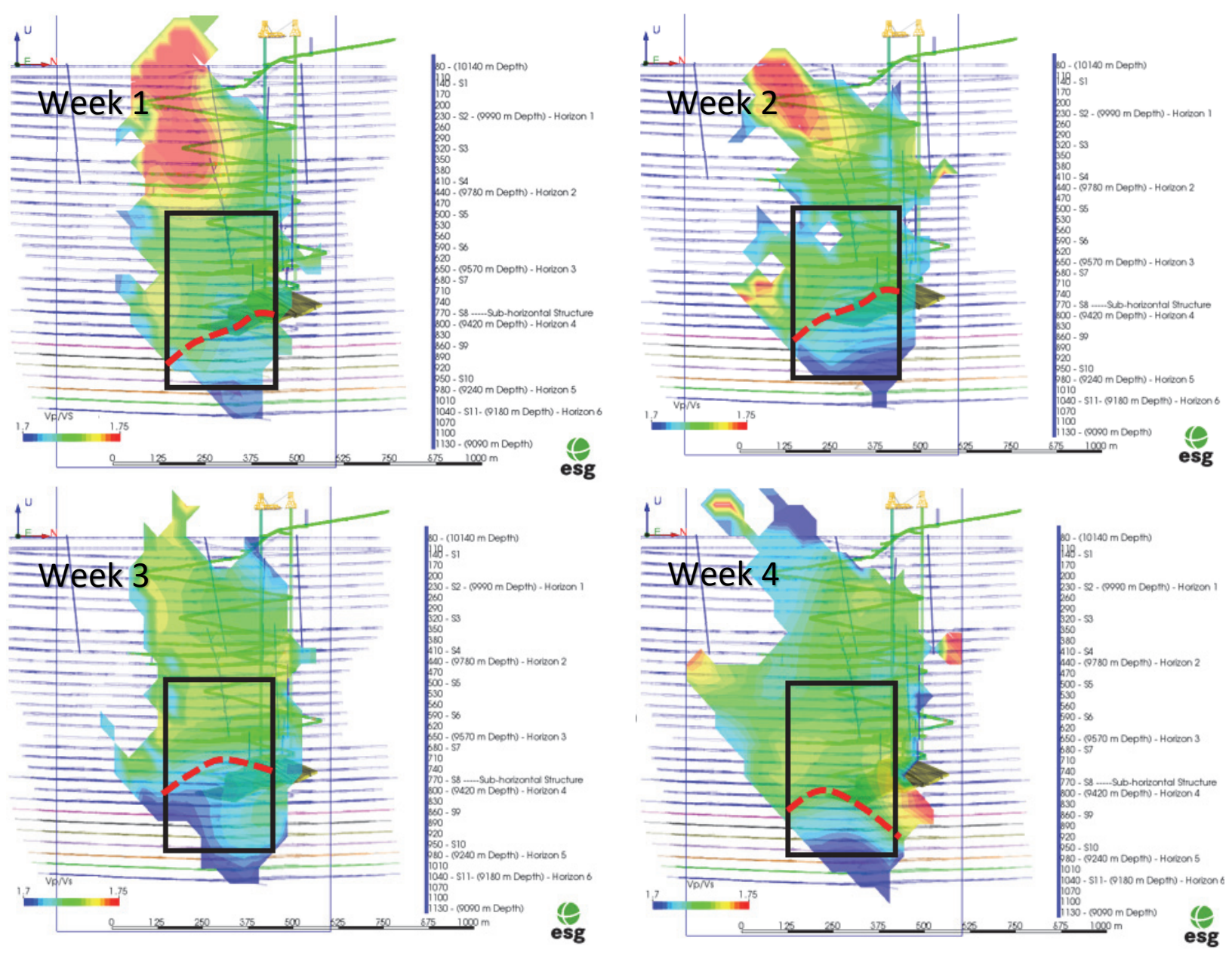

Figure 6 The $V_{p} / V_{s}$ (proxy to Poisson's ratio) tomography results for four consecutive weeks. The red line shows the consistent pattern around the large sub-horizontal structure (higher $V_{p} / V_{s}$ values in the upper part and lower in the lower part). Rectangles show the areas with better resolutions

\section{$5 \quad$ Seismic hazard assessment}

\subsection{Statistical seismic parameters}

The variations in the apparent stress, $E_{s} / E_{p}$ ratio and tomography indicate that there is a marked difference in the rock mass in horizon 5 and below the sub-horizontal structure. There is a sharp contrast in the seismic activity above and below this zone. As a result, the potential for strain bursts and rock bursts in each formation is different. To quantify the characteristics of each horizon, empirical probability distributions were estimated for several different statistical seismic hazard parameters. These were evaluated before and after a large seismic event has occurred. In this paper, the results for the seismic strain rate are discussed, as well as the potential for large aftershocks. The end-product is a probability table which can assist in establishing re-entry protocols.

Seismic strain rate is one of several statistical seismic parameters used to quantify the seismic response. These seismic hazard parameters are part of ESG Solutions's SeisWatch software (and described in Malek \& Leslie 2006, and Table 1) to quantify different aspects of the seismic response following a large event. The seismic strain rate uses a group of seismic events and estimates the total moment of all the events, the volume enclosed by the events and the timing of the events. The seismic strain rate serves as a proxy for the strain rate due to coseismic deformation. 
Table 1 The statistical seismic parameters used by SeisWatch to assess the current level of seismic hazard

\begin{tabular}{ll}
\hline SeisWatch parameters & Proportional to \\
\hline Event decay rate & Event number and time \\
Seismic work (and work rate) & Strength and time \\
Clustering & Location and time \\
Strain rate & Strength, location and time \\
Event level frequency & Depth, location and time \\
\hline
\end{tabular}

An example of this data is shown in Figure 7 for the seismic strain rate calculated for the entire mine. A large event occurs (time denoted with the first vertical dashed line), and there is an increase in the seismic strain rate. This increase is caused by the large event, and the resulting aftershocks keep the seismic strain rate elevated for several hours. Once the perturbation to the stress field from the large event has dissipated, the seismic strain rate lowers, indicating that the rate and intensity of the seismicity has returned to its typical levels. This typical behaviour is referred to as the background. The time required for the seismic strain rate (estimated for the entire mine) to return to the background level is estimated (shown in the Figure 7 as $\tau_{s t}$ ). In real-time, several of these statistical parameters (from Table 1) can be evaluated to help assess the current level of seismic hazard throughout the mine.

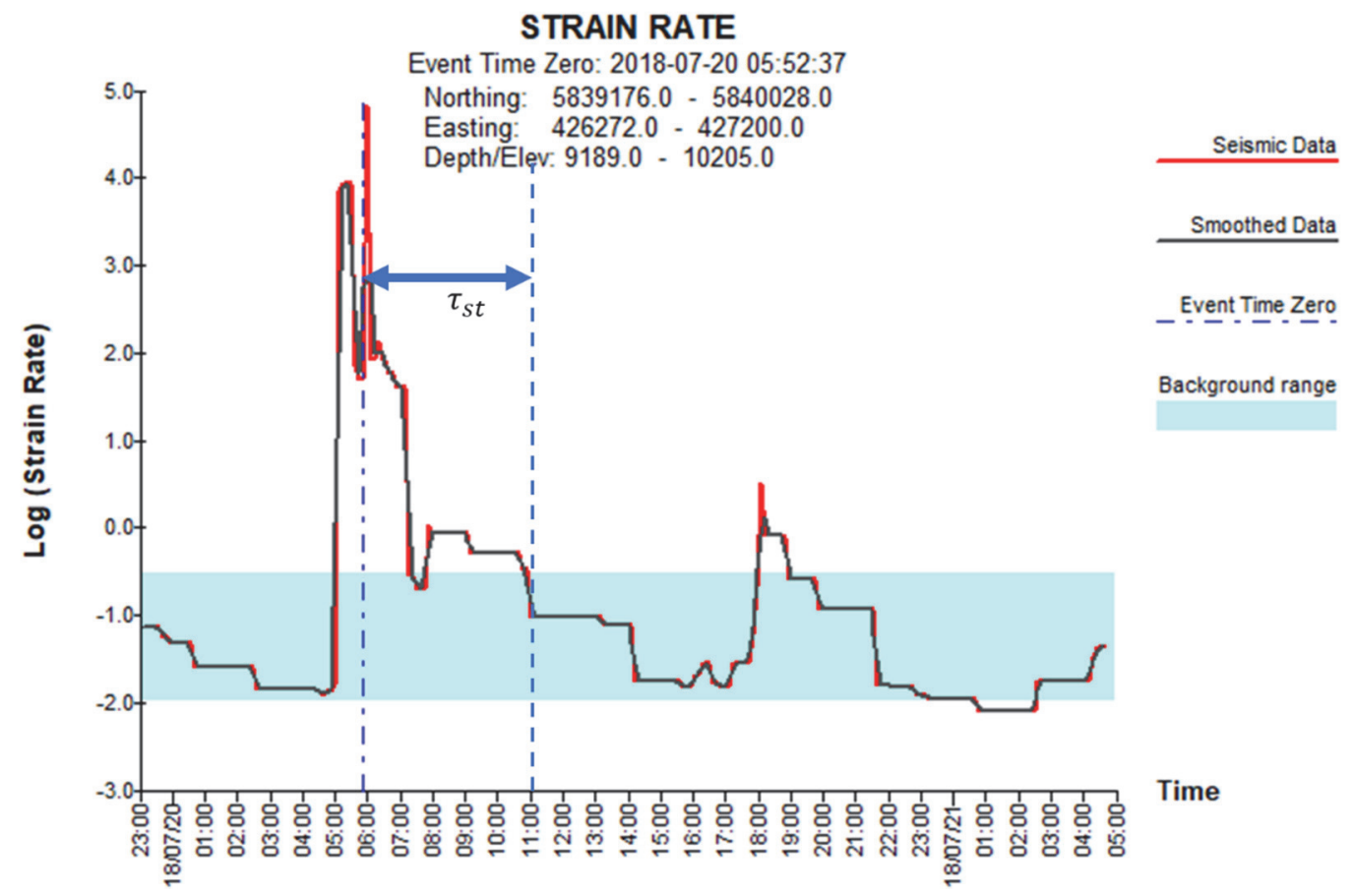

Figure 7 The seismic strain rate. The first vertical line (dashed and dotted) represents the time of a large event. The second line (dashed) shows the time period over which the signal decays back to the background level (shown as a light blue shaded region)

\subsection{Empirical seismic hazard assessment}

Using nearly three years of seismic data recorded at Éléonore, the seismic response following large events in horizons 4 and 5 are compared quantitatively. For each large event in horizons 4 and 5, (shown in blue and orange respectively in Figure 8), a background level of seismic activity is estimated using all events within $300 \mathrm{~m}$ of the large event and 48 hours of data before the large event occurred. For the purposes of this study, a large event is defined as an event with $M_{w} \geq 0.7$. 
Using an automated algorithm, the time required to return to the more stable state is estimated for the seismic strain rate, seismic work, clustering coefficient, and event rate. Only the results for the seismic strain rate are discussed here in order to outline the concepts and methods.

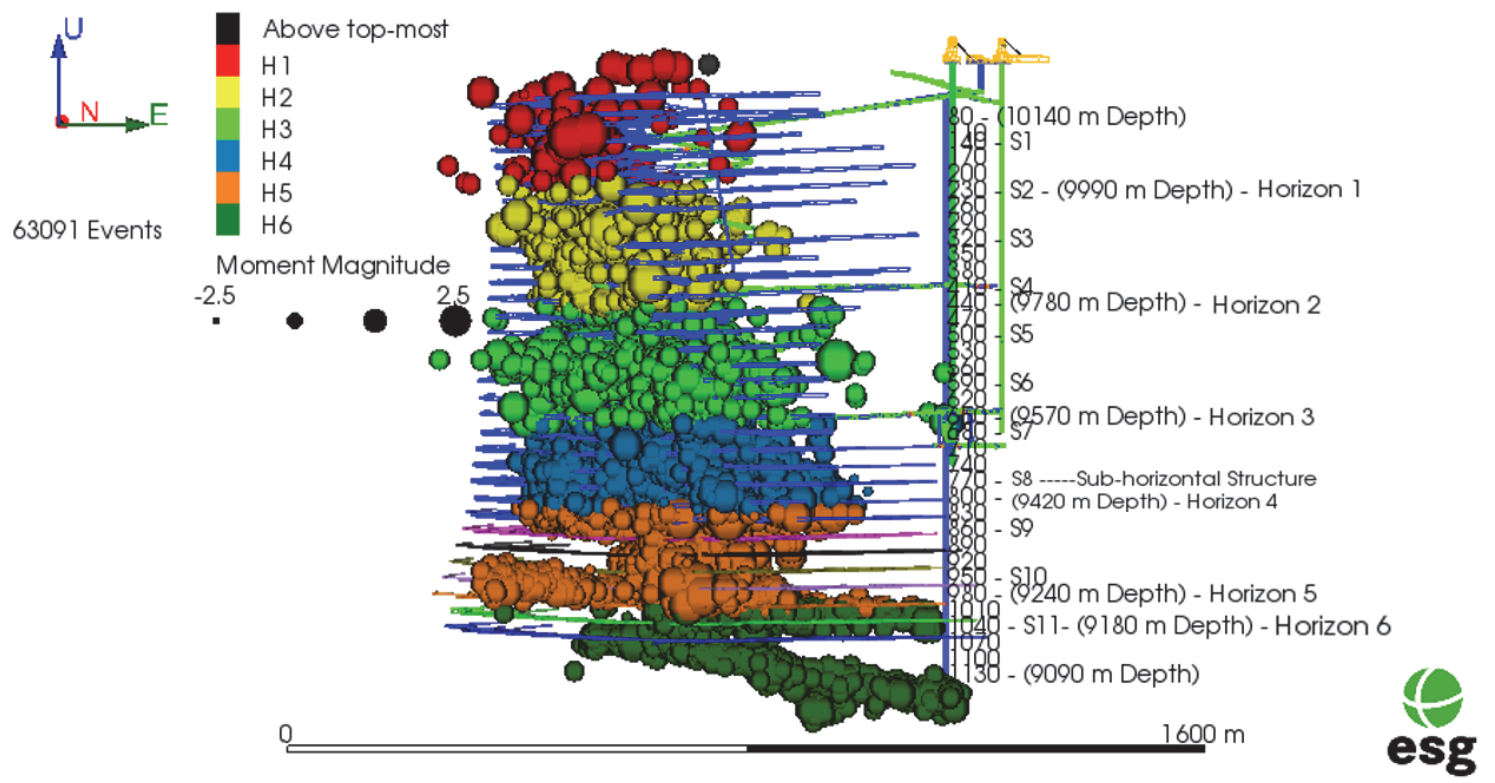

Figure 8 The location and magnitude of the events used in the seismic hazard assessment

The time required to return to the background behaviour in horizons 4 and 5 are shown in Figures $9(\mathrm{a})$ and (b) for the seismic strain rate. For each large event within the horizon, the time required to return to the background (more stable behaviour) varies. Horizon 5 has $28 \%$ more large events than horizon 4 , but there are some similarities in the seismic response. In both horizons, stable conditions return within 10-20 hours following most of the large events. However, sometimes it takes more than 70 hours to return to the background. Within horizon 5, when a large event occurs, it can sometimes take far longer to return to the background behaviour.

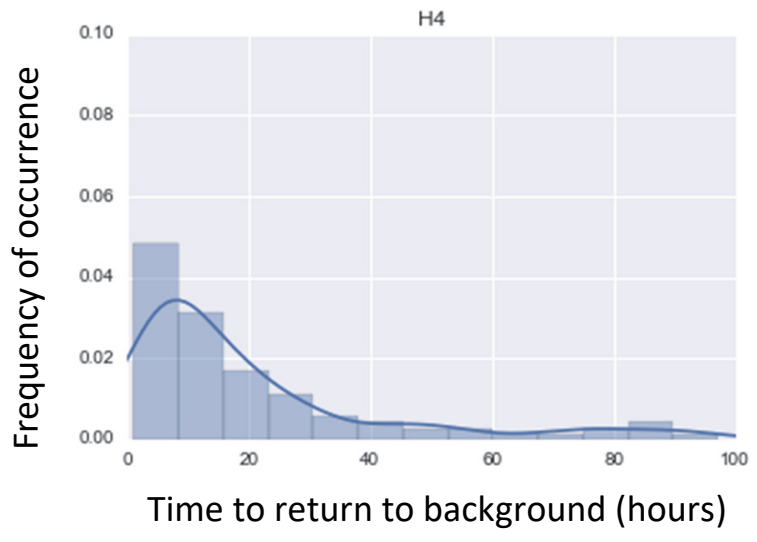

(a)

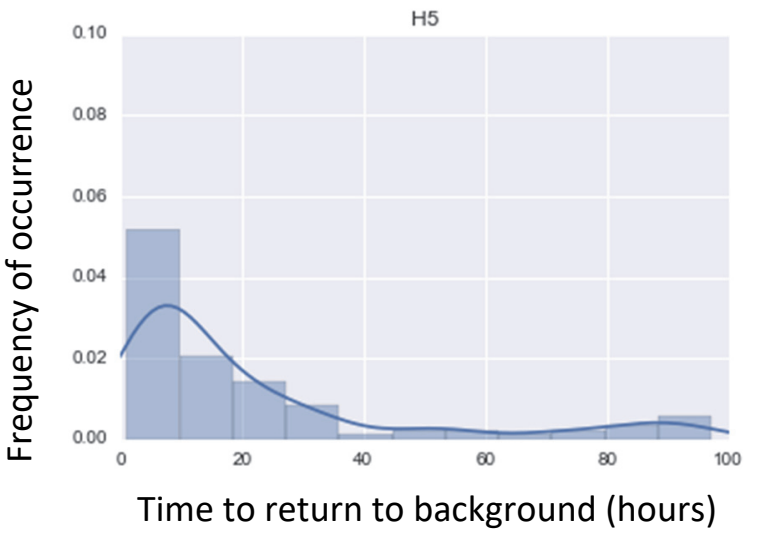

(b)

Figure 9 The distribution of times required to return to the background behaviour for the seismic strain rate. Blue rectangles show the normalised histograms. The smooth blue line is an estimate of the empirical probability density function

To assign a likelihood for each of the observations, the empirical data is transformed into a cumulative distribution function. From this, an estimate of the probability of returning to the background in less than XX hours is made. These results are shown in Table 2 and show very different results for horizons 4 and 5 . 
If a large event occurs within horizon 4 , then $75 \%$ of the time, the seismic strain rate returns to the background within 17 hours. Within horizon 5, it takes 29 hours to return to the background (for $75 \%$ of the observed data). Occasionally, the seismic strain rate takes a very long time to return to the background, with only $90 \%$ of the observed large event responses returning within 74 hours for horizon 5 . Within horizon 4 , where the apparent stress is lower, $90 \%$ of the observed responses had returned to typical behaviour within 32 hours.

Table 2 The probability of the seismic strain rate returning to the previous background level within a number of hours

\begin{tabular}{lcc}
\hline & \multicolumn{2}{c}{ Hours within } \\
\hline Probability (\%) & In horizon 4 & In horizon 5 \\
\hline 25 & 4 & 7 \\
50 & 8 & 11 \\
75 & 17 & 29 \\
90 & 32 & 74 \\
95 & 66 & 85 \\
\hline
\end{tabular}

\subsection{Probability of large aftershocks}

Following a large event within the mine, one potential hazard is the occurrence of a large aftershock. To evaluate the potential hazard within Éléonore, the time between successive large events was evaluated for each large event in horizons 4 and 5.

For 100 hours following each large event, we searched the catalogue to determine if a large aftershock (here defined as $M_{w} \geq 0.5$ ) occurred within $300 \mathrm{~m}$. If an aftershock did occur within this window, then the time of occurrence was recorded. These results are shown in Figure 10.

Horizon 5 had far more large aftershocks than horizon 4, particularly around 24 hours after the original event.

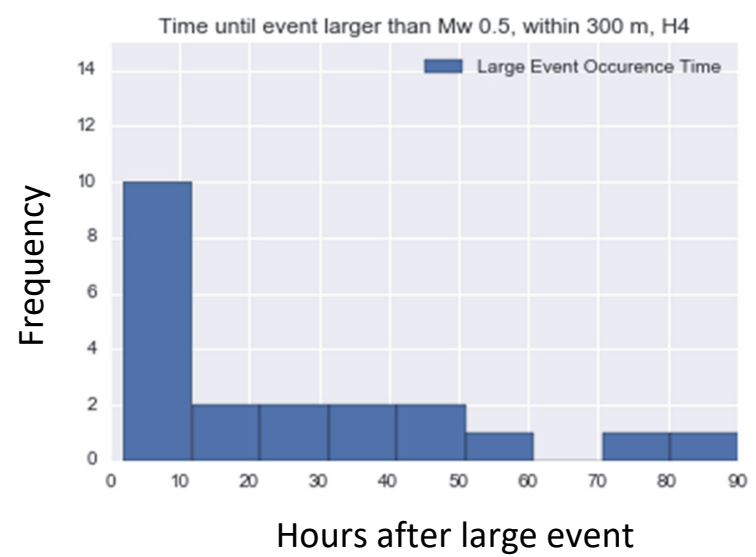

(a)

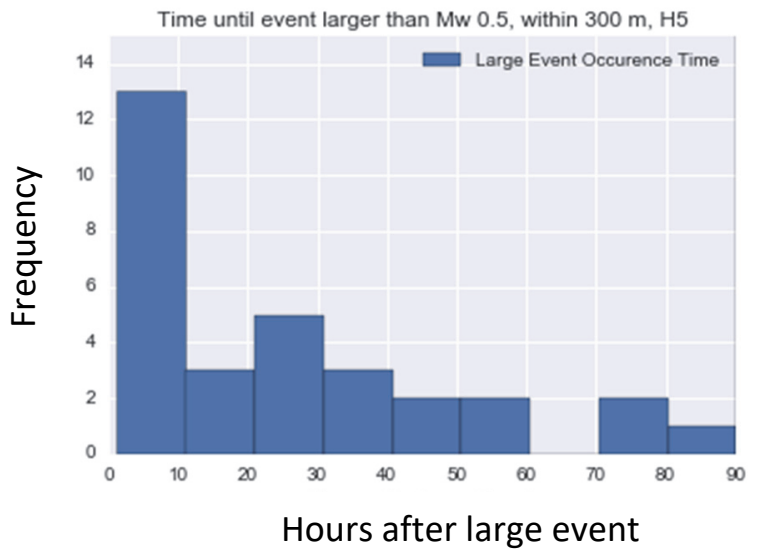

(b)

Figure 10 Histogram of the average time between successive large events in (a) horizon 4 and (b) horizon 5. There is a marked increase in the number of large events in horizon 5 
To further quantify this behaviour, the probability of aftershocks occurring within XX hours was estimated. These results are outlined in Table 3. From these results, large aftershocks only occur after $15 \%$ of the large events in horizon 4 , and $17 \%$ of the large events in horizon 5 . The timing of the aftershocks varies for each formation. For horizon 4, there is a $15 \%$ chance that another large event will occur within 81 hours following one large event. In horizon 5, the events occur much more rapidly, with a 15\% chance of another large event occurring within 52 hours.

Table 3 The probability of one large event occurring within different time intervals following a large seismic event. There is a longer time interval between large events in horizon 4

\begin{tabular}{lccc}
\hline & \multicolumn{2}{c}{ Hours within } \\
\hline Probability of a large event occurring (\%) & In horizon 4 & In horizon 5 \\
\hline 5 & 3 & 3 \\
10 & 32 & 28 \\
15 & 81 & 52 \\
\hline
\end{tabular}

\section{Conclusion}

Over three years, seismic source parameters were compared to the mine geotechnical data and travel-time tomography. The intention was to customise the re-entry protocol and seismic hazard tool according to the rock mass behaviour changes in different mine horizons.

Seismic source parameter analysis showed a distinctive behaviour in the deeper horizon 5 , in comparison to the shallower horizons, in which lower-magnitude seismic events had a higher $E_{s} / E_{p}$ and static stress drop in horizon 5. Also, blast and event cumulative apparent stress trends, assisted in tracking the consistency and variations of the stress field. In addition, travel-time tomography utilising microseismic data was used to validate the changes in the P- and S-wave velocities associated with mine horizons. The three mentioned analyses were in good match with each other and suggest a sharp contrast in the rock mass and stress across the sub-horizontal structure. The results were used as inputs for seismic hazard assessment.

The seismic hazard assessment quantified the differences in the potential for seismic hazard in horizons 4 and 5, where the largest contrast in source parameters and tomography was observed. To describe the method, one of five parameters was used to highlight differences in the two horizons. In practice, a combination of many of these statistical seismic parameters are used to evaluate the potential for seismic hazard and aid in the development of re-entry times and the analysis can be repeated to highlight changes.

With the seismic strain rate, sharp differences in the response after a large seismic event $\left(M_{w} \geq 0.7\right)$ were observed. Following a large event in horizon 4, stable conditions returned within 17 hours in $75 \%$ of the cases studied. In horizon 5, there were more large events, and a longer time period following these events was needed before the seismic response returned to more typical behaviour within 29 hours for $75 \%$ of the large events.

The likelihood of an aftershock occurring was nearly identical for both horizons; roughly $15 \%$ of the time an aftershock with $\mathrm{M}_{\mathrm{w}} \geq 0.5$ occurred within 100 hours after a large event. The timing of these aftershocks varied between the formations. Aftershocks occurred much sooner in horizon 5 than in horizon 4.

It is important to note that, since the variation in the stress gradient in depth is higher than east and north directions, the procedure described cannot be applied to the east and north directions of the mine, unless the selected events in different sections are in the same elevation. This issue can be solved by dividing the interested volume into blocks and comparing the blocks individually. 
Several methods using seismic data were applied to highlight and describe conditions within a mine. Seismic source parameter analysis indicated variations in stress and source processes. Tomography highlighted areas with different seismic velocities (and stress). Seismic hazard analysis provided quantifiable estimates of how those differences resulted in different responses following a large seismic event. Combined, these techniques allow for the assessment of the potential for seismic hazard within the mine and can easily be repeated to track changes in stress, strain and the potential for seismic hazard as mining progresses. With this information, more-accurate and customised re-entry protocols can be developed.

\section{Acknowledgement}

We sincerely thank Goldcorp Inc. for making available the site investigation data, seismic data, and technical documents associated with Éléonore mine.

\section{References}

Abolfazlzadeh, Y \& Hudyma, MR 2016, 'Identifying and describing a seismogenic zone in a sublevel caving mine', Rock Mechanics and Rock Engineering, vol. 49, no. 9, pp. 1-17, https://doi.org/10.1007/s00603-016-1017-x

Brune, JN 1970, 'Tectonic stress and the spectra of seismic shear waves from earthquakes', Journal of Geophysical Research, vol. 75, no. 26, pp. 4997-5009.

Brown, L \& Hudyma, M 2017b, 'Identifying local stress increase using a relative apparent stress ratio for populations of mining-induced seismic events', Canadian Geotechnical Journal, vol. 54, no. 1, pp. 128-137.

Carusone, O \& Hudyma, M 2017, 'Variations in apparent stress and energy index as indicators of stress and yielding around excavations', in M Hudyma \& Y Potvin (eds), Proceedings of the First International Conference on Underground Mining Technology, Australian Centre for Geomechanics, Perth, pp. 205-218.

Dahlen, FA 1974, 'On the ratio of P-wave to S-wave corner frequencies for shallow earthquake sources', Bulletin of Seismological Society of America, vol. 64, pp. 1159-1180.

Goldcorp Inc. 2012, internal technical report, Éléonore operations, Goldcorp Inc., Quebec.

Hudyma, MR 2008, Analysis and Interpretation of Clusters of Seismic Events in Mines, PhD thesis, The University of Western Australia, Perth.

Malek, F \& Leslie, IS 2006, 'Using seismic data for rockburst re-entry protocol at Inco's copper cliff north mine', Proceedings of the 41st US Symposium on Rock Mechanics (USRMS): 50 Years of Rock Mechanics - Landmarks and Future Challenges. ", American Rock Mechanics Association, Alexandria.

Mine Design Engineering 2017, Factual Report \#1015-F1704-01: Geotechnical Mapping and Site Characterization, Goldcorp internal report, Mine Design Engineering, Kingston.

Ravenelle, JF, Dubé, \& B Malo, M 2010, Insights on the Geology of the World-class Roberto Gold Deposit, Éléonore Property, James Bay Area, Quebec, Geological Survey of Canada, Ottawa.

Rawlinson, N \& Sambridge, M 2003, 'Seismic traveltime tomography of the crust and lithosphere', Advances in Geophysics, vol. 46, pp. 81-198.

Vallejos, J \& McKinnon, SD 2011, 'Correlations between mining and seismicity for re-entry protocol development', International Journal of Rock Mechanics and Mining Sciences, vol. 48, no. 4, pp. 616-615.

Woodward, KR \& Tierney, SR 2017, 'Seismic hazard estimation using databases with bimodal frequency-magnitude behaviour', in M Hudyma \& Y Potvin (eds), Proceedings of the First International Conference on Underground Mining Technology, Australian Centre for Geomechanics, Perth, pp. 219-232.

Wesseloo, J 2014, 'Evaluation of the spatial variation of b-value', The journal of the Southern African Institute of Mining and Metallurgy, vol. 114, pp. 823-828.

Wyss, M \& Brune, JN 1986, 'Seismic moment, stress and source dimensions for earthquakes in the California-Nevada region', Journal of Geophysical Research, vol. 73, pp. 4681-4694.

Young, DP 2012, Energy Variations in Mining-Induced Seismic Events Using Apparent Stress, MSc thesis, Laurentian University, Sudbury. 